\title{
La collecte des « Dainas » par Krišjānis Barons
}

\author{
Julien Gueslin
}

\section{(2) OpenEdition}

Journals

Édition électronique

URL : http://journals.openedition.org/rbnu/688

DOI : 10.4000/rbnu.688

ISSN : 2679-6104

\section{Éditeur}

Bibliothèque nationale et universitaire de Strasbourg

\section{Édition imprimée}

Date de publication : 1 novembre 2017

Pagination : 68-71

ISBN : 9782859230678

ISSN : 2109-2761

\section{Référence électronique}

Julien Gueslin, «La collecte des «Dainas » par Krišjānis Barons », La Revue de la BNU [En ligne], 16 | 2017, mis en ligne le 01 juillet 2019, consulté le 11 décembre 2020. URL : http://

journals.openedition.org/rbnu/688; DOI : https://doi.org/10.4000/rbnu.688

\section{(C) $10(0$}

La Revue de la BNU est mise à disposition selon les termes de la Licence Creative Commons Attribution - Pas d'Utilisation Commerciale - Partage dans les Mêmes Conditions 4.0 International. 
Chansons $N$ ationales Letrevionos

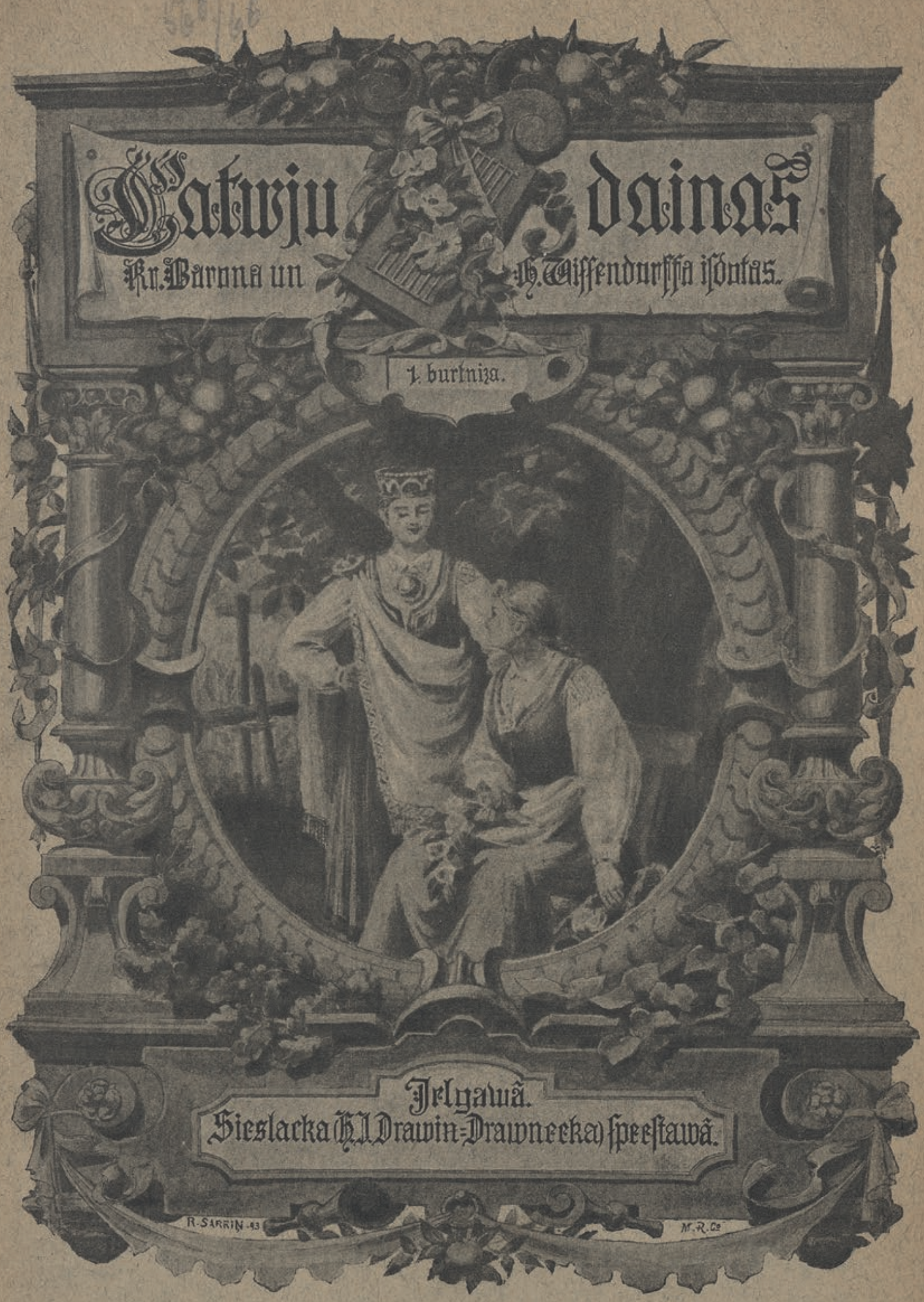




\section{LETTONIE \\ LA COLLECTE DES «DAINAS » PAR KRIŠJĀNIS BARONS}

PAR JULIEN GUESLIN

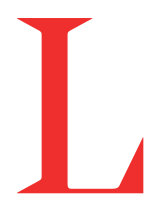

orsque Krišjānis Barons meurt en 1923, cinq ans après la proclamation de l'indépendance lettonne, c'est tout un peuple qui fait des funérailles nationales à celui que certains surnomment l'« Homère letton ». Mais Barons a été moins un barde ou le créateur d'une épopée nationale qu'un scientifique qui a édité, en six tomes et huit volumes, des milliers de textes de chansons populaires et contribué à forger l'identité nationale lettonne. Barons est l'un des acteurs de la renaissance nationale lettonne de la seconde moitié du $19^{\text {e }}$ siècle. Celle-ci vise, sur le modèle des autres nations européennes, à affirmer le droit à l'existence et au respect d'une petite nationalité. Le peuple letton a vécu, depuis le $13^{\text {e }}$ siècle, sous la domination d'élites germanophones qui ont réussi à maintenir leur domination sociale malgré les nombreuses vicissitudes politiques ayant affecté l'espace baltique (guerres de Livonie, luttes entre la Russie, la Suède et la Pologne débouchant finalement sur le triomphe, au $18^{\mathrm{e}}$ siècle, de l'empire tsariste).
Les anciens serfs lettons (le servage n'est aboli qu'au début du $19^{\mathrm{e}}$ siècle) sont-ils une «nation sans histoire " (ni littérature), comme aimeraient le croire une forte majorité des grands propriétaires et de la bourgeoisie urbaine germano-baltes ? Ces derniers évoquent le rôle des colons allemands (Porte-Glaive, Teutoniques, commerçants, clercs) ayant selon eux civilisé et christianisé (par l'épée) une région barbare. Si la Réforme débouche sur la nécessité d'étudier la langue lettonne (avec les premiers extraits de chansons publiés dans les grammaires éditées, comme celle de Stender au $18^{\mathrm{e}}$ siècle), c'est la génération romantique qui met en valeur l'idée de "Volksgeist " et la nécessité de valoriser les traditions populaires de chaque peuple. Herder lui-même, qui séjourne à Riga dans les années 1760 , semble avoir été fortement influencé par des contacts qui lui ont permis de découvrir la beauté, la simplicité et le dépouillement de cette poésie rurale. Ce sont les pasteurs germano-baltes, les plus au contact des populations paysannes, qui vont favoriser la publication des premiers recueils de chansons (Bergmann, Wahr en 1807-1808, Büttner en 1844). 
Dans la seconde moitié du $19^{\mathrm{e}}$ siècle, l'émergence d'une jeune bourgeoisie lettonne change la donne. Elle veut donner ses lettres de noblesse à ce qu'elle voit désormais comme une nation devant redécouvrir son histoire. Contrairement aux autres nations, on ne peut exalter le souvenir d'un État ancien ou d'une culture " classique ». Il faut contrecarrer les stéréotypes en valorisant les traditions ancestrales, que ce soit par l'archéologie (afin de montrer l'évolution des tribus lettonnes avant l'arrivée des chevaliers allemands) ou par l'étude des traditions populaires. La multiplication des chorales, le début des grands festivals de chants (le premier a lieu en 1873) commencent cependant à faire la renommée de cette "nation chantante ». Dans des campagnes déjà très alphabétisées, où le développement de l'instruction et des sociétés savantes s'accélère, un des premiers devoirs assignés aux personnes éduquées est de récolter et de noter le plus grand nombre possible de chansons caractérisant chaque terroir. Le travail de collecte et de rassemblement des chants populaires devient un des premiers éléments d'une identité nationale collective élaborée par cette " communauté imaginaire " (au sens de B. Anderson) en voie de consolidation. Celle-ci se structure par cette recherche, qui est aussi un moyen très concret de diffuser un sentiment national en gestation auprès des masses et ainsi de les impliquer.

Barons s'inscrit dans la suite de ce mouvement. Né en 1835 en Courlande, il a la chance de pouvoir faire des études. Il étudie les mathématiques et l'astronomie à l'université de Dorpat (aujourd'hui Tartu en Estonie, où se rassemblait la jeunesse des provinces baltes). Il fait partie de cette première génération qui refuse de se fondre dans le moule de la culture germanique dominante, seul moyen alors de pouvoir espérer progresser dans la hiérarchie sociale, et il défend ses origines face à la condescendance des élites.

Il collabore à un des premiers journaux lettons, les Pēterburgas Avīzes, dirigé par Krišjānis Valdemārs, une des premières grandes figures du mouvement national letton. Après l'arrêt de la publication, suite aux pressions des milieux germano-baltes, il devient précepteur en Russie mais continue à être un acteur important du mouvement national. C'est à la fin des années 1870 qu'il se décide à suivre et à coordonner de manière systématique l'entreprise de recueil des chansons populaires. Véritable savant " de cabinet ", il va consacrer le reste de sa vie à classer et à rédiger toutes les chansons notées et envoyées avec leurs très nombreuses variantes locales et régionales (au total plus de 200000 textes et plus de 35000 chansons). Il élabore même une armoire spéciale servant de cartothèque pour toutes les chansons recensées. Ce meuble précieusement conservé à Riga deviendra vite une véritable icône nationale ; il a été inscrit par l'UNESCO au patrimoine mondial de l'humanité. À la différence des précédents recueils, Barons entreprend donc un véritable travail scientifique afin de donner une unité au corpus et de permettre de comparer les nombreuses variantes. Il se conforme à un principe de classement dit " ethnographique ", rassemblant les chants d'après les circonstances de la vie humaine (en particulier le mariage, avec trois volumes) et le moment où ils sont chantés (fêtes, en particulier les grandes fêtes de Jāni et Līgo célébrant le solstice d'été les 23 et 24 juin, et liées aux rites de fertilité). Les variantes (avec lieu et date de collecte) sont regroupées. Grâce au soutien de Lettons anonymes, mais aussi de mécènes (comme $\mathrm{H}$. Wissendorff) et de sociétés savantes russes (comme l'Académie des sciences de Saint-Pétersbourg), eux-mêmes intéressés et impliqués activement dans ce travail ethnographique, il peut mener à bout son entreprise. Commencé en 1894 avec la publication du premier volume à Mitau (Jelgava), ce travail monumental (édité par la suite à Saint-Pétersbourg) ne sera achevé qu'en 1915. Revenu à Riga en 1893 pour mieux terminer son travail, Barons accède de son vivant à un véritable rôle de patriarche national, son âge vénérable lui permettant de voir une Lettonie indépendante sans avoir à se mêler aux luttes politiques.

Les chansons populaires sont pour l'essentiel des quatrains, comparant un moment de la vie naturelle avec la vie humaine, et comportent rarement plus de trois thèmes. Malgré leur importance pour la vie nationale, elles n'évoquent que très rarement des moments historiques ou ce qui aurait pu être la vie des tribus lettonnes dans un passé ancien. Faute de données sur les conditions et le moment de leur création, elles semblent surtout se rapporter à la vie menée à la période médiévale et au début de la Renaissance (peu de mentions du luthéranisme, de la domination russe ou des livres imprimés). Mêmes les chansons de caractère mytholo- 
gique et évoquant certaines divinités semblent dater de cette époque où, malgré un christianisme de façade, le paganisme restait largement répandu. Évoquant le cycle naturel de la vie, de la nature comme des hommes, les "dainas " (chants) sont perçus comme une véritable "Bible du peuple " reflétant l'âme lettonne, où la tristesse et le sérieux des motifs évoqués ne débouchent sur aucun désespoir, haine ou toute autre forme de sentimentalisme, mais plutôt sur une foi en la vie et la volonté de dépasser les épreuves, une opposition passive à la domination des seigneurs, voire une certaine ironie.

Il ne faut pas oublier non plus que pour les intellectuels et linguistes lettons qui eurent également pour tâche de créer une véritable langue nationale normalisée, les dainas ont été aussi une source abondante pour l'étude de la morphologie ou de la phonétique, avec la présence de nombreux mots absents des dictionnaires lettons existant au $19^{\mathrm{e}}$ siècle.

\section{Quelques exemples de dainas recueillis par Barons :}

En chantant, je suis né, en chantant, j'ai grandi,

En chantant j'ai vécu ma vie ;

En chantant mon âme est arrivée

Dans le jardin du fils de Dieu.

(Vitols, p. 13)

Pourquoi, Laima, m'as-tu choisi

Un chemin de vie aussi raboteux ?

Est-ce que tu ne pouvais pas

M'asseoir sur un siège de seigneur?

(Vitols, p. 59)

Moi seul obéissais en chantant

Aux ordres de maîtres cruels,

Car j'étanchais ainsi mes larmes

Avec une petite chanson

(Vitols, p. 61)

Tiré de Dainas : poèmes lettons / traduits et présentés par Nadine Vitols Dixon, Montpellier, L'Archange Minotaure, 2004 